Neurodegeneration: The Molecular Pathology of Dementia ANd Movement Disorders. Second Edition 2011. Edited by Dennis W. Dickson, Roy O. Weller. Published by WileyBlackwell. 477 pages. C\$290 approx.

\section{Rated tristror}

This book describes neurodegenerative diseases from the perspective of molecular pathology, covering a broad range of topics in dementia and movement disorders. This book is distinct from other books in that it is structured using molecular pathology mechanisms, as opposed to clinical syndrome or anatomical distribution. There are eight parts: Introduction to basic mechanisms of neurodegeneration, Alzheimer's disease, Tauopathies, Synucleinopathies, Trinucleotide Repeat Disorders, Prion Disorders. In the last two sections, the authors discuss Frontotemporal Lobar Degeneration/ALS/MND and other neurodegenerative disorders.

The first section is useful, the authors

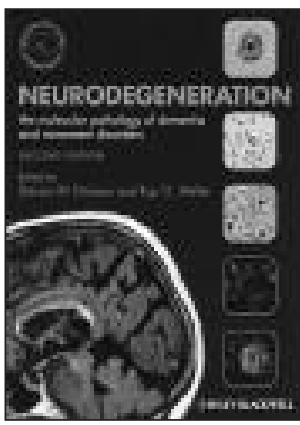
discussing the basic mechanisms of cell death, protein abnormalities and genetics relevant to various neurodegenerative diseases. The other sections begin with an introduction to orient the readers, followed by chapters on different diseases within the section (such as PD, DLB, MSA under Synucleinopathies). In each chapter, there is coverage of clinical features, genetics, neuroimaging, macroscopy, histopathology, biochemistry, pathogenesis and future directions. The authors generally follow that step by step format, which makes for easy reading. The histopathological pictures are very clear and the tables are systematic.

However, if someone is looking for knowledge about clinical syndromes, this book is not the appropriate reference. The clinical component is general and brief, not going into specific details. The therapeutic part is also short or even non-existent in some chapters. In some topics, the differential diagnosis is done by clinical presentation, but in others it is done by histopathological features.

Overall, this textbook provides useful and systematic information on the basic mechanisms of neurodegenerative diseases. It will prove useful for resident trainees, neurologists and pathologists.
Parkinson's Disease - Non-Motor and Non-Dopaminergic Features. 2011. Edited by C. Warren Olanow, Fabrizio Stocchi, Anthony E. Lang. Published by Wiley-Blackwell. 474 pages. C\$205 approx.

\section{Rated tortartor}

"It's a page turner!" That's how I summarized the book to my non-neurologist husband when I was writing the review. I initially tried to skim read the book to get an overview but immediately got sucked into reading much more detail than intended. My husband thought that this was clear evidence of neurologists really being "nerdologists" (a term introduced by another neurologist spouse) but for me as a movement disorder neurologist the book very successfully fills an important gap in the Parkinson's literature: An overview of the field beyond dopamine and motor features. In the levodopa era we treat motor symptoms fairly successfully but now non-motor and non-dopaminergic symptoms often dominate clinical practice, the patients' and their families' life and increasingly research in the field.

The editor and author list reads like an international A-team of experts with strong Canadian contributions. The book comprehensively covers the many aspects of non-dopaminergic/non-motor PD symptoms from neuropathology, anatomy, animal models, imaging, rating scales, and premotor symptoms to the future development of therapies. It then dedicates the vast majority of chapters to the discussion of individual symptoms and their etiology, epidemiology, clinical presentation, management and current research. Individual symptoms discussed include dementia (four chapters), neuropsychiatric symptoms (psychosis, depression, anxiety, impulsive and compulsive behaviours), sleep disorders (three chapters), autonomic dysfunction (gastroinestinal and swallowing disturbance, urinary problems, orthostatic hypotension, sexual dysfunction), sensory symptoms (mainly olfaction, pain and paraesthesias), restless legs syndrome and akathisia, speech and voice problems, gait and postural symptoms, orthopedic complications and miscellaneous symptoms including fatigue (I wish there was more material than three paragraphs on this important problem, though!). In addition, there are overviews of the medical and surgical treatment approaches and a more basic science chapter on effects of exercise focusing on animal studies. I would have welcomed a chapter on non-motor/nondopaminergic symptoms in genetically determined Parkinsonism but I am sure there will be future editions. Figures in the chapters are in black and white with some color plates in the middle of the book; text boxes are successfully used to summarize important points.

In the age of online literature searches the value of the book lies in providing a comprehensive summary of the field of non-motor and non-dopaminergic symptoms in PD, integrating the clinical and scientific perspective, current knowledge and implications for future research. It extensively covers clinical management aspects 
with many useful recommendations and should thus be very helpful to neurologists and geriatricians. Residents should have access to the book in their departments. After all, patients benefit from having "nerdologists" as their physicians and neurologists tend to love good books - this is one of them.

Silke Appel-Cresswell Vancouver, British Columbia, Canada

Seven Aneurysms: Tenets and Techniques for Clipping. 2011. By Michael T. Lawton. Published by Thieme Medical Publishers, Inc. 224 pages. C\$160 approx.

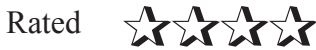

This is a well conceived and very well illustrated book that will appeal to the beginner vascular neurosurgeon and to the general neurosurgeon looking for an introduction or a refresher on aneurysm surgery prior to a specific case. The book's main sections are organized according to the frequency of aneurysms encountered, e.g. the MCA, PComm, AComm locations. The intraoperative photographs are exquisitely detailed, and in a nice step-by-step fashion.

An attractive volume, it reflects the personal experience of one neurosurgeon, and is written in a "how I do it" format. It has already served as a useful starting-off point for our own neurosurgery residents and neurovascular fellows. I found the technical discussions, while excellent, to be a bit dogmatic, e.g. concerning type of microscope, the endorsement of surgical mouthpieces, and the eschewing of brain retraction, induced hypotension and lumbar drainage. I would have liked more detail concerning the use of specific microneurosurgical intruments e.g. the \#6 Rhoton. The book would also have been strengthened with more detail concerning alternative approaches, e.g.

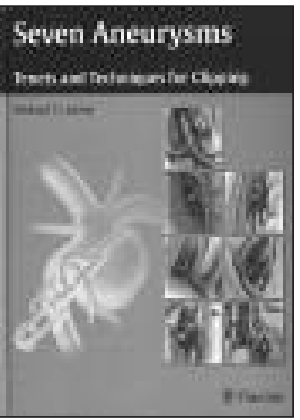
the subtemporal approach to the upper basilar and temporal approach to the posterior cerebral artery. The references were somewhat sparse, and largely comprised articles written or cowritten by the author.

Notwithstanding these concerns, the book serves it purpose well. Since many aneurysms are now treated by endovascular means, the surgical experience gained by residents and fellows has diminished. Thus a monograph such as this one, emphasizing the basics, and with lots of photographs, is both timely and useful.

Stephen P. Lownie London Health Sciences Centre London, Ontario, Canada 